\title{
Jet physics at HERA
}

\author{
Claudia Glasman* \\ (On behalf of the H1 and ZEUS Collaborations) \\ Universidad Autónoma de Madrid \\ E-mail: claudia.glasman@uam.es
}

The latest results on jet physics from the ZEUS and H1 Collaborations at HERA are presented. The measurements include inclusive-jet, dijet and three-jet cross sections in the photoproduction and deep inelastic ep scattering regimes. The measured cross sections have the potential to constrain the proton and photon parton distribution functions, especially the gluon density. A QCD analysis of the measurements have allowed the extraction of very precise values of the strong coupling and its energy-scale dependence.

35th International Conference of High Energy Physics - ICHEP2010,

July 22-28, 2010

Paris France

${ }^{*}$ Speaker. 


\section{Introduction}

The HERA ep collider is a very suitable environment to test QCD in hadronic-induced reactions. During the last decade, jet physics at HERA has reached such a high level of precision as to make stringent tests of QCD and precision measurements of the strong coupling, $\alpha_{s}$. Jet cross sections are also suitable as constraints of parton distribution functions (PDFs).

Jet production at HERA proceeds via the deep inelastic ep scattering (DIS) and photoproduction processes. Jet production in neutral current (NC) DIS at $\mathscr{O}\left(\alpha_{s}\right)$ in the Breit frame, in which the photon and the proton collide head on, proceeds via the boson-gluon fusion and QCD Compton processes. The jet-production cross-section prediction can be written as the convolution of the proton PDFs and the partonic cross section, which is calculable in perturbative QCD (pQCD). In photoproduction, two processes contribute at lowest order: the direct processes, in which the photon interacts with the partons of the proton as a point-like particle, and the resolved processes, in which the photon interacts with the partons of the proton via its partonic structure. The crosssection prediction for jet photoproduction can be written as the convolution of the flux of photons from the electron beam, the photon and proton PDFs and the partonic cross section. Thus, measurements of jet cross sections in NC DIS and photoproduction allow tests of the structure of the photon and the proton, tests of $\mathrm{pQCD}$ and determinations of $\alpha_{s}$.

\section{Constraints on the PDFs from jet cross sections}

Jet cross sections, both in the NC DIS and photoproduction regimes are directly sensitive to the gluon component in the proton through the boson-gluon fusion process and can test the PDFs extracted from other channels or constrain the gluon density if incorporated, together with structure function data, in the fits to extract the PDFs. The ZEUS Collaboration has included jet data in the fits to extract the proton PDFs [1]; the result was an improvement of the determination of the gluon density by up to a factor of two in the mid- to high- $x$ region, where $x$ is the fraction of the proton momentum taken by the interacting parton.

To ascertain the potential of the new jet cross-section measurements to constrain the gluon density, the predicted gluon fraction and the theoretical uncertainties were studied in the phasespace region of the measurements [2]. Figure 1 shows examples for dijet cross sections in the region $125<Q^{2}<20000 \mathrm{GeV}^{2}$, where $Q^{2}$ is the virtuality of the exchanged photon. The predicted gluon fraction is bigger than $75 \%$ at low $Q^{2}$ and decreases to $\sim 50 \%$ for $Q^{2} \approx 500 \mathrm{GeV}^{2}$. The PDF uncertainty is large and the uncertainty from higher orders is small in regions of phase space where the gluon fraction is still sizeable; thus, the dijet data have the potential to constrain the gluon density.

The H1 Collaboration has measured dijet cross sections in NC DIS for $5<Q^{2}<100 \mathrm{GeV}^{2}$ and $0.2<y<0.7$ [3], where $y$ is the inelasticity. The jets were identified using the $k_{T}$ cluster algorithm [4] in the longitudinally invariant inclusive mode [5] in the Breit Frame and required to have jet transverse momentum in the Breit frame $P_{T}>5 \mathrm{GeV}$ and $-1<\eta_{\mathrm{LAB}}^{\text {jet }}<2.5$, where $\eta_{\mathrm{LAB}}^{\text {jet }}$ is the jet pseudorapidity in the laboratory frame; the invariant mass of the dijet system, $M^{\mathrm{jj}}$, was restricted to above $18 \mathrm{GeV}$ to remove the regions of phase space where the theoretical calculations have limitations. Figures $2 \mathrm{a}$ and $2 \mathrm{~b}$ show the measured dijet cross sections as functions of the 
(a)

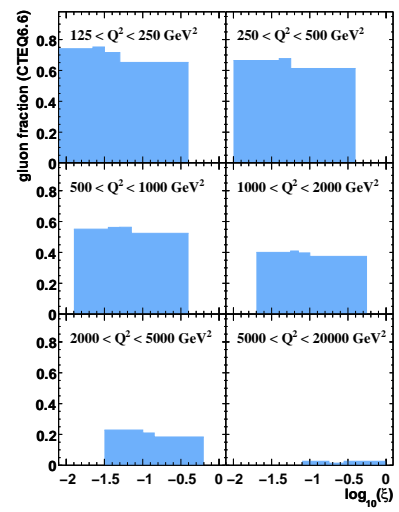

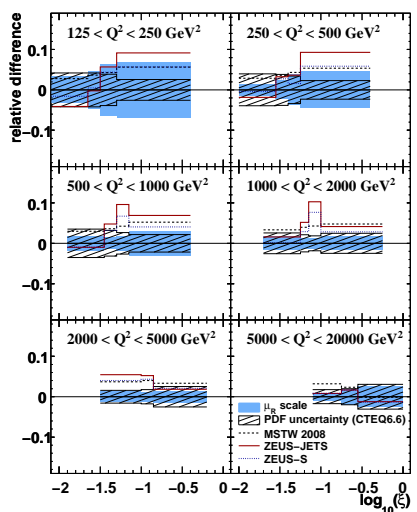

(b)

Figure 1: (a) Predicted gluon fraction and (b) theoretical uncertainties as functions of $\xi$ in different regions of $Q^{2}$.
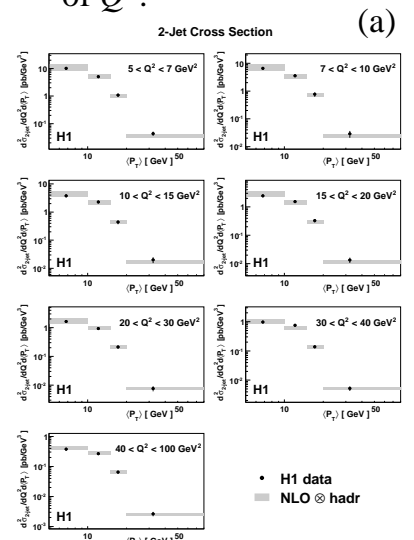
- H1 data
NLO $\otimes$ had

(a)
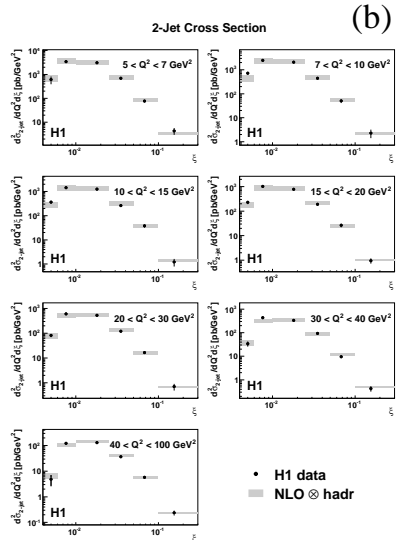

(b)

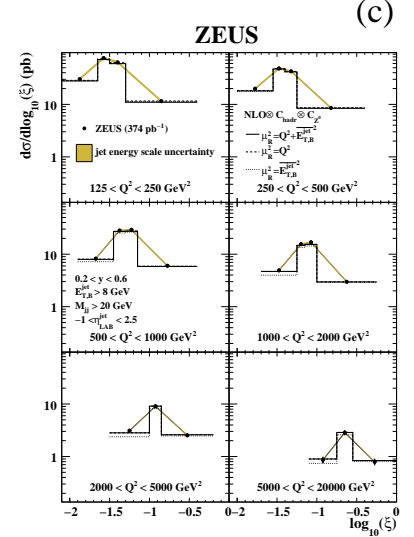

(c)
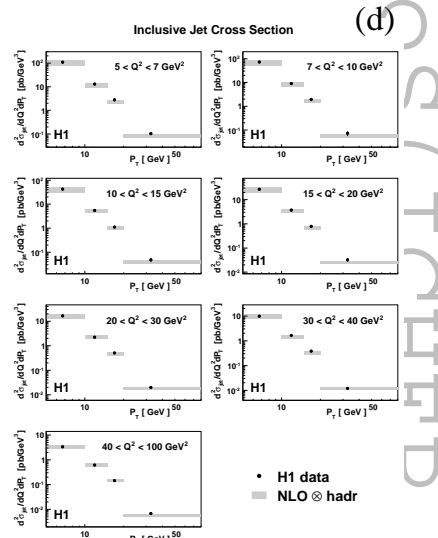

Figure 2: Dijet cross sections as functions of (a) $\left\langle P_{T}\right\rangle$ and (b) $\xi$ in different regions of $Q^{2}$; (c) Dijet cross sections as functions of $\xi$ in different regions of $Q^{2}$; (d) Inclusive-jet cross sections as functions of $P_{T}$ in different regions of $Q^{2}$.

average $P_{T}$ of the two jets, $\left\langle P_{T}\right\rangle$, and of $\xi=x_{\mathrm{Bj}}\left(1+\left(M^{\mathrm{jj}}\right)^{2} / Q^{2}\right)$, which is an estimator of the momentum carried by the struck parton and $x_{\mathrm{Bj}}$ is the Bjorken scaling variable, in different regions of $Q^{2}$, respectively. The data are very precise with uncorrelated uncertainties of $\sim \pm 5(15) \%$ at low (high) $\left\langle P_{T}\right\rangle$; the jet energy scale uncertainty, which has been reduced to $\pm 2 \%$, gives a contribution of $\pm 5(15) \%$ at low (high) $\left\langle P_{T}\right\rangle$. The uncorrelated (correlated) uncertainties are $\sim \pm 6 \%(\sim \pm 5 \%)$ as a function of $\xi$. The next-to-leading-order (NLO) QCD predictions were computed using the program NLOJET++ [6] with renormalisation, $\mu_{R}$, and factorisation, $\mu_{F}$, scales set to $\mu_{R}^{2}=\mu_{F}^{2}=$ $\left(Q^{2}+\left\langle P_{T}\right\rangle^{2}\right) / 2$. The CTEQ6.5M [7] sets were used for the proton PDFs and the calculations were corrected for hadronisation effects. The measurements are well described by the NLO predictions. The gluon fraction is very large at these low values of $Q^{2}$; however, the theoretical uncertainties, dominated by the terms beyond NLO, are also very large. Next-to-next-to-leading-order (NNLO) predictions are needed to take full advantage of these high-precision data to constrain the PDFs.

The ZEUS Collaboration has measured the dijet cross sections [2] for jets of $E_{T, \mathrm{~B}}^{\mathrm{jet}}>8 \mathrm{GeV}$, where $E_{T, \mathrm{~B}}^{\mathrm{jet}}$ is the jet transverse energy in the Breit frame, as functions of $\xi$ in the range $125<$ $Q^{2}<20000 \mathrm{GeV}^{2}$ (see Fig. 2c). The experimental uncorrelated uncertainties are $\sim \pm 2(10) \%$ at low (high) $Q^{2}$; the jet energy scale uncertainty, which has been reduced to $\pm 1 \%$, gives a contribution of \pm 5 (2)\% at low (high) $Q^{2}$. The NLO calculations were computed using the program NLOJET++ with $\mu_{R}^{2}=Q^{2}+\left(\overline{E_{T, \mathrm{~B}}^{\mathrm{jet}}}\right)^{2}$ and $\mu_{F}=Q$. The CTEQ6.6 [8] sets were used for the proton 
(a)

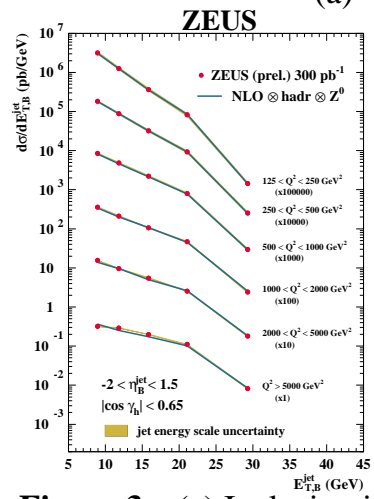

(b)

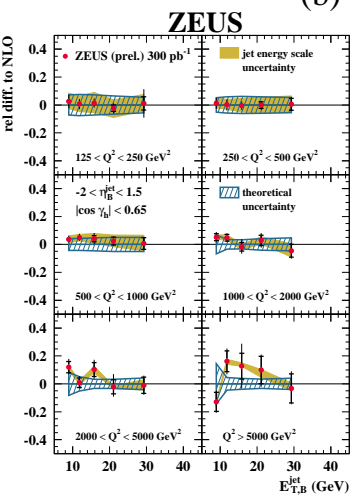

(c)

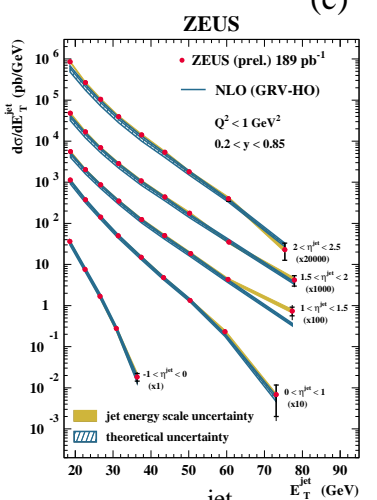

(d)

Figure 3: (a) Inclusive-jet cross sections in NC DIS as functions of $E_{T B \text { B }}^{\text {jet }}$ in different regions of $Q^{2}$; (b) the relative difference of the data in (a) to the NLO calculations; (c) Inclusive-jet cross sections in photoproduction as functions of $E_{T}^{\text {jet }}$ in different regions of $\eta^{\text {jet }}$; (d) the relative difference of the data in (c) to the NLO calculations.

PDFs. The calculations, which were corrected for hadronisation and $Z^{0}$ effects, describe well the measurements. At $Q^{2} \approx 500 \mathrm{GeV}^{2}$, there is still a sizeable gluon contribution (more than $50 \%$ ) and the theoretical uncertainties from higher orders are reduced to $\pm 6 \%$ at these high values of $Q^{2}$; therefore, the sensitivity to the PDFs is large.

Inclusive-jet cross-section measurements can also constrain the PDFs. The H1 Collaboration has measured [3] inclusive-jet cross sections as functions of $P_{T}$ in different regions of $Q^{2}$ for $5<$ $Q^{2}<100 \mathrm{GeV}^{2}$ (see Fig. 2d). The $P_{T}$ spectrum gets harder as $Q^{2}$ increases. The measurements are well described by the NLO calculations. As in the case of dijet production, the gluon fraction is large in this region of phase space, but the theoretical uncertainty from higher orders is $\sim \pm 30 \%$; thus, NNLO calculations are needed to take full advantage of these high-precision data.

The ZEUS Collaboration has measured [9] inclusive-jet cross sections for $Q^{2}>125 \mathrm{GeV}^{2}$ and $E_{T, \mathrm{~B}}^{\mathrm{jet}}>8 \mathrm{GeV}$ in NC DIS. Figures $3 \mathrm{a}$ and $3 \mathrm{~b}$ show the cross sections as functions of $E_{T, \mathrm{~B}}^{\mathrm{jet}}$ in different regions of $Q^{2}$. The measured cross sections show that the $E_{T, \mathrm{~B}}^{\mathrm{jet}}$ spectrum becomes harder as $Q^{2}$ increases. The experimental uncorrelated uncertainties are $\sim \pm 3(10) \%$ at low (high) $Q^{2}$; the jet energy scale uncertainty, which has been reduced to $\pm 1 \%$, gives a contribution of \pm 5 (2)\% at low (high) $Q^{2}$. The NLO calculations were computed using the program DisENT [10] with $\mu_{R}=E_{T, \mathrm{~B}}^{\mathrm{jet}}$ and $\mu_{F}=Q$. The ZEUS-S [11] sets were used for the proton PDFs. The calculations, which were corrected for hadronisation and $Z^{0}$ effects, describe the data very well in the whole measured range.

The ZEUS Collaboration has measured [12] the inclusive-jet cross sections in photoproduction for $E_{T}^{\mathrm{jet}}>17 \mathrm{GeV}$, where $E_{T}^{\mathrm{jet}}$ is the jet transverse energy in the laboratory frame. Figures $3 \mathrm{c}$ and $3 \mathrm{~d}$ show the inclusive-jet cross sections as functions of $E_{T}^{\text {jet }}$ in different regions of jet pseudorapidity. The measured spectra get harder as $\eta^{\text {jet }}$ increases. The NLO calculations, computed using the program by Klasen, Kleinwort and Kramer [13] with $\mu_{R}=\mu_{F}=E_{T}^{\mathrm{jet}}$, based on different proton and photon PDF sets and corrected for hadronisation effects, are compared to the data. The NLO calculations give a good description of the data except for low $E_{T}^{\text {jet }}$ and high $\eta^{\text {jet }}$. The theoretical uncertainties are dominated by terms beyond NLO, but at high (low) $E_{T}^{\text {jet }}$ the contributions from terms beyond NLO and the proton (photon) PDFs are of the same order. Therefore, these cross sections have the potential to constrain both the photon and the proton PDFs. 
(a)

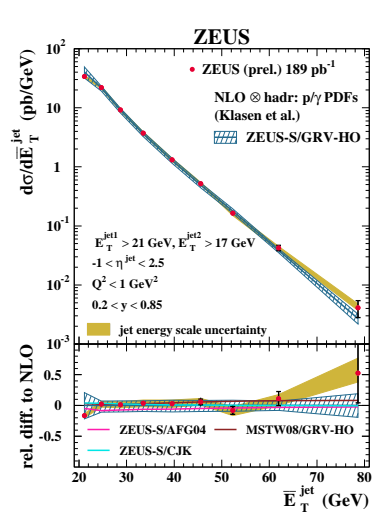

(b)

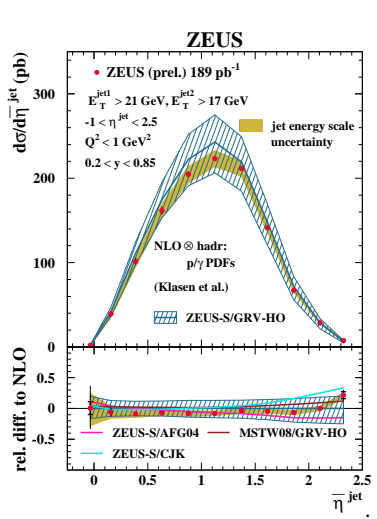

Claudia Glasman

(c)

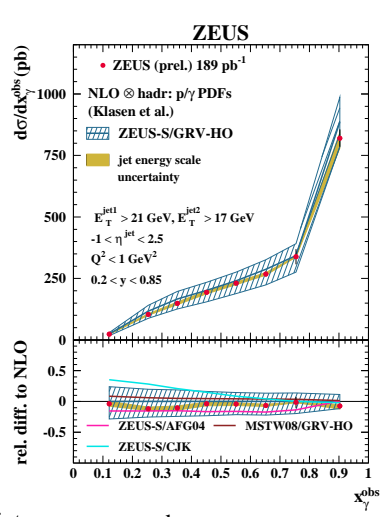

Figure 4: Dijet cross sections as functions of (a) $\bar{E}_{T}^{\mathrm{jet}}$, (b) $\bar{\eta}^{\mathrm{jet}}$ and (c) $x_{\gamma}^{\mathrm{obs}}$.

Dijet cross sections as functions of the averaged $E_{T}^{\text {jet }}, \bar{E}_{T}^{\mathrm{jet}}$, and $\eta^{\mathrm{jet}}, \bar{\eta}^{\mathrm{jet}}$, and the fraction of the photon energy invested in the dijet system, $x_{\gamma}^{\text {obs }}$, were measured [14] by ZEUS (see Fig. 4). The cross sections are well described by the NLO predictions. The comparison of the measurements to the predictions based on different PDFs show the sensitivity of these cross sections to both the photon (at high $\eta^{\text {jet }}$ and low $x_{\gamma}^{\text {obs }}$ ) and the proton (at high $E_{T}^{\text {jet }}$ ) PDFs.

\section{Tests of QCD and determination of $\alpha_{s}$}

Tests of pQCD have been made by the H1 Collaboration based on the measurements of inclusive-jet, dijet and trijet cross sections at low $Q^{2}$ [3]. Figure 5a shows the measurements as functions of $Q^{2}$ and $P_{T}$. The data have very small correlated and uncorrelated uncertainties. The NLO calculations describe the measurements well; however, the predictions exhibit very large uncertainties from higher orders, $\sim \pm 30 \%$ at the lowest $Q^{2}$ values and $\pm 10 \%$ at higher $Q^{2}$; other uncertainties are relatively small. This shows the need for NNLO calculations. These measurements are directly sensitive to the value of $\alpha_{s}$ with small experimental uncertainties. The energy-scale dependence of the coupling was determined by extracting $\alpha_{s}$ from the measured jet cross sections. Figure $5 \mathrm{~b}$ shows the results as a function of the scale, which are in good agreement with the predicted running of $\alpha_{s}$ with small experimental uncertainties. A value of $\alpha_{s}\left(M_{Z}\right)$ was determined by a simultaneous fit to the measurements: $\alpha_{s}\left(M_{Z}\right)=0.1160 \pm 0.0014$ (exp. $)_{-0.0079}^{+0.0094}$ (th.). This determination has very small experimental uncertainties, but the theoretical uncertainties, dominated by the terms beyond NLO, are very large. In order to reduce the theoretical uncertainties, $\alpha_{s}\left(M_{Z}\right)$ was determined from the measured trijet to dijet ratio (see Figs. $5 \mathrm{c}$ and $5 \mathrm{~d}$ ). In this ratio, the correlated uncertainties cancel and a determination of $\alpha_{s}\left(M_{Z}\right)$ with smaller theoretical uncertainties at low $Q^{2}$ was obtained; however, the experimental uncertainties increase. Thus, to extract a more precise value of $\alpha_{s}\left(M_{Z}\right)$ from these measurements, NNLO calculations are needed.

Normalised jet cross sections [15] were measured by $\mathrm{H} 1$ for $150<Q^{2}<15000 \mathrm{GeV}^{2}$. The inclusive-jet cross sections normalised to the inclusive $\mathrm{NC}$ cross section in the same region of phase space are shown in Fig. 6a as functions of $P_{T}$ in different regions of $Q^{2}$. The normalised inclusivejet cross section may be interpreted as the average jet multiplicity produced in NC DIS, which increases with $Q^{2}$ since the available phase space opens up with $Q^{2}$. The same feature is observed in the dijet and trijet normalised cross sections. In all cases, the NLO calculations give a good description of the data with small experimental and theoretical uncertainties. These cross sections 
(a)

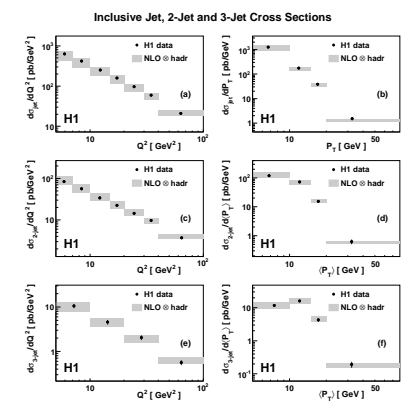

(b)

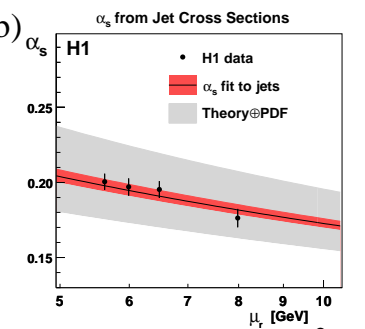

(c)

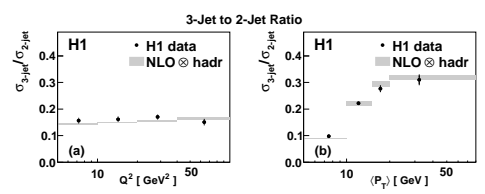

(d)

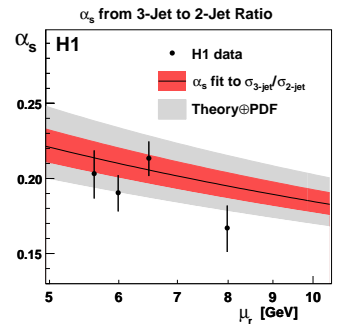

Figure 5: (a) Multijet cross sections as functions of $Q^{2}$ and $\left\langle P_{T}\right\rangle$; (b) $\alpha_{s}$ extracted from jet cross sections as a function of the scale; (c) trijet to dijet ratios as functions of $Q^{2}$ and $\left\langle P_{T}\right\rangle$; (d) $\alpha_{s}$ extracted from the ratio as a function of the scale.

(a)
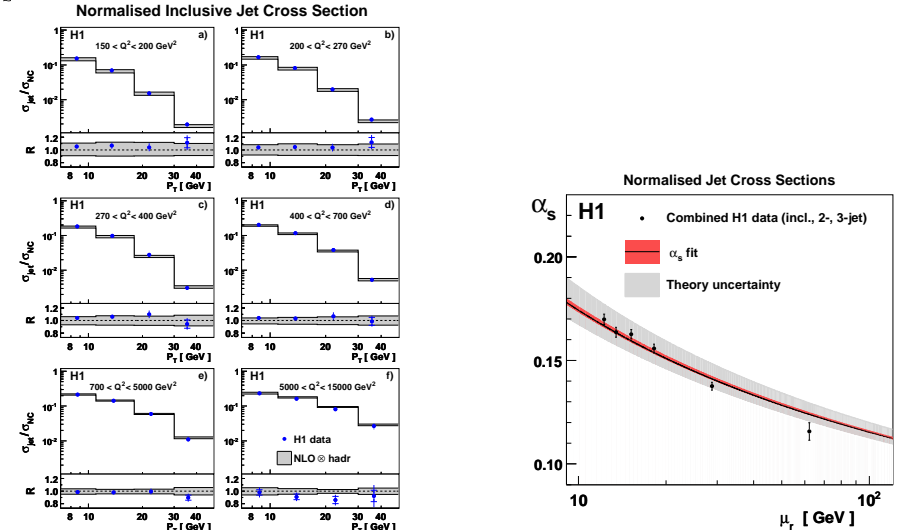

Figure 6: (a) Normalised inclusive-jet cross sections as functions of $P_{T}$ in different regions of $Q^{2}$; (b) $\alpha_{s}$ extracted from jet cross sections as a function of the scale.

are also sensitive to the value of $\alpha_{s}$. The energy-scale dependence of the coupling was determined by extracting $\alpha_{s}$ from the measured normalised jet cross sections at high $Q^{2}$. Figure $6 \mathrm{~b}$ shows the results, which are in good agreement with the predicted running of $\alpha_{s}$ with small experimental and theoretical uncertainties. These reduction of uncertainties with respect to the measurements at low $Q^{2}$ come from extracting $\alpha_{s}$ from normalised cross sections, since the correlated uncertainties cancel in the ratio, and from performing the extraction at higher $Q^{2}$, since the theoretical uncertainties decrease. A value of $\alpha_{s}\left(M_{Z}\right)$ was extracted from a simultaneous fit to all the measurements at high $Q^{2}: \alpha_{s}\left(M_{Z}\right)=0.1168 \pm 0.0007$ (exp. $)_{-0.0034}^{+0.0049}$ (th.). The precision is limited by the theoretical uncertainties; thus, NNLO calculations would yield an improved determination.

The inclusive-jet cross sections as functions of $Q^{2}$ and $E_{T, B}^{\mathrm{jet}}$ [9] were measured by ZEUS for $Q^{2}>125 \mathrm{GeV}^{2}$ (see Fig. 7). The measured cross sections decrease by more than five (three) orders of magnitude within the measured $Q^{2}\left(E_{T, \mathrm{~B}}^{\mathrm{jet}}\right)$ range and have small experimental uncertainties. The theoretical uncertainties are also small and dominated by the terms beyond NLO, which is smaller than $5 \%$ for $Q^{2}>250 \mathrm{GeV}^{2}$. The NLO calculations describe very well the measured distributions. These cross sections are directly sensitive to $\alpha_{s}$ and present small experimental and theoretical uncertainties; therefore, they are particularly suited to determine this fundamental parameter. The energy-scale dependence of the coupling was determined by extracting $\alpha_{s}$ from the measured cross section as a function of $E_{T, \mathrm{~B}}^{\mathrm{jet}}(\mathrm{see} F \mathrm{Fig} .7 \mathrm{c}$ ). The results are in very good agreement 
(a)

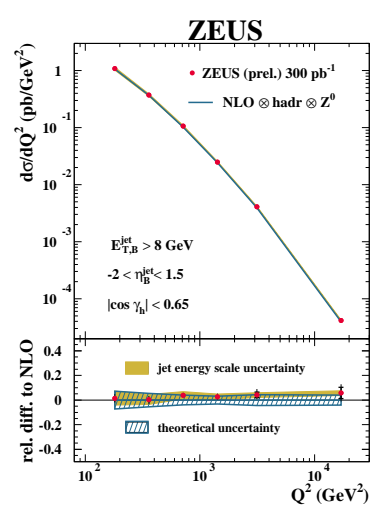

(b)

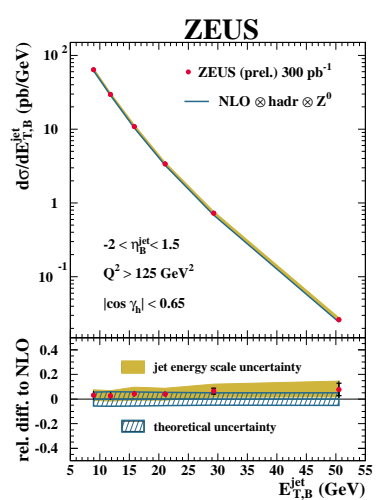

(c)

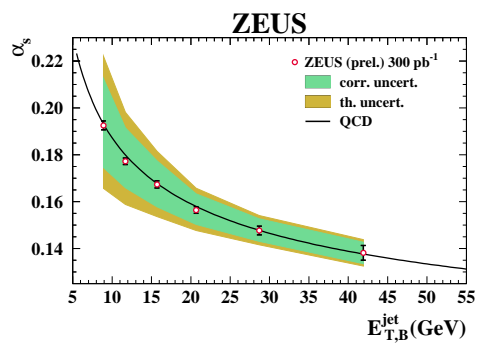

Figure 7: Inclusive-jet cross sections as functions of (a) $Q^{2, \mathrm{a}, \mathrm{Ge}}$ (b) $E_{T, \mathrm{~B}}^{\mathrm{jet}}$; (c) $\alpha_{s}$ as a function of $E_{T, \mathrm{~B}}^{\mathrm{jet}}$.

(a)

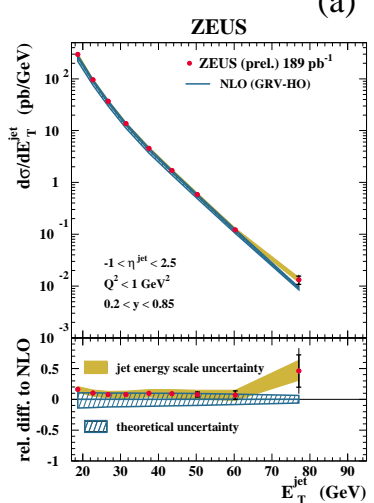

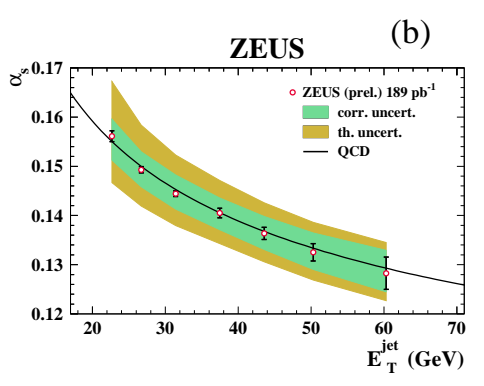

(c)

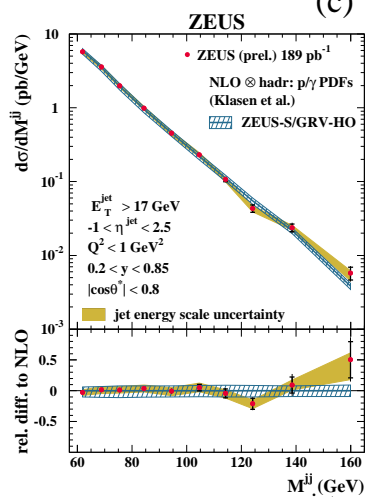

(d)

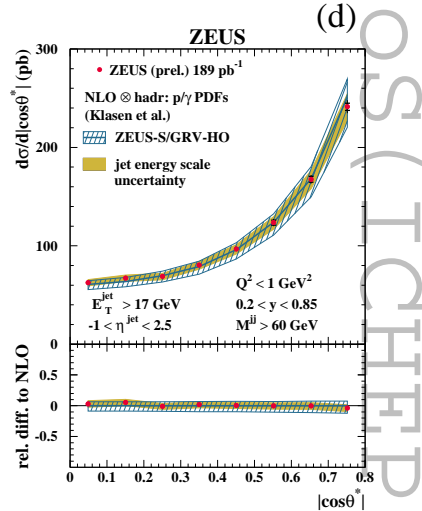

Figure 8: (a) Inclusive-jet cross section as a function of $E_{T}^{\text {jet }}$; (b) $\alpha_{s}$ as a function of $E_{T}^{\text {jet }}$. Dijet cross sections as functions of (c) $M^{\mathrm{jj}}$ and (d) $\left|\cos \theta^{*}\right|$.

with the predicted running of $\alpha_{s}$ over a large range in $E_{T, \mathrm{~B}}^{\mathrm{jet}}$. A value of $\alpha_{s}\left(M_{Z}\right)$ was determined for $Q^{2}>500 \mathrm{GeV}^{2}: \alpha_{s}\left(M_{Z}\right)=0.1208_{-0.0032}^{+0.0037}$ (exp.) ${ }_{-0.0022}^{+0.0022}$ (th.). This determination has very small theoretical uncertainty and the precision is limited by the experimental uncertainty.

Tests of pQCD were also done in the photoproduction regime. Figure 8a shows the inclusivejet cross section as a function of $E_{T}^{\text {jet }}$ [12]. The measured cross section decreases by more than four orders of magnitude within the measured range. The experimental and theoretical uncertainties are small. The NLO calculation gives a good description of the data. This cross section is also sensitive to the value of $\alpha_{s}$. The energy-scale dependence of the coupling was determined for $21<E_{T}^{\text {jet }}<71$ $\mathrm{GeV}$; the results are shown in Fig. $8 \mathrm{~b}$ and are in good agreement with the predicted running of $\alpha_{s}$ over a large range of $E_{T}^{\mathrm{jet}}$. The value of $\alpha_{s}\left(M_{Z}\right)$ determined from this measurement is: $\alpha_{s}\left(M_{Z}\right)=$ $0.1208{ }_{-0.0023}^{+0.0024}$ (exp.) ${ }_{-0.0033}^{+0.0044}$ (th.), whose precision is limited by the theoretical uncertainty.

There is a discrepancy between NLO and data at low $E_{T}^{\text {jet }}$ and high $\eta^{\text {jet }}[12]$, which can be seen clearly in the comparison between data and theory as a function of $\eta^{\text {jet }}$ in Fig. 9a. This discrepancy could be attributed to an inadequacy of the photon PDFs or to non-perturbative effects. The NLO calculations based on the AFG04 [16] photon PDFs give lower predictions compared to the default, which uses the GRV-HO [17] sets, and the predictions are higher, closer to the data, when using the CJK [18] PDFs. Non-perturbative effects were taken into account using Monte Carlo simulations which include multiple interactions (see Fig. 9b); the effect of the non-perturbative contribution is to increase the jet rate at high $\eta^{\text {jet}}$, where the discrepancy between data and NLO is largest. Also, the disagreement between data and NLO disappears when increasing $E_{T}^{\mathrm{jet}}$, as can be seen in Fig. 9c, 
(a)

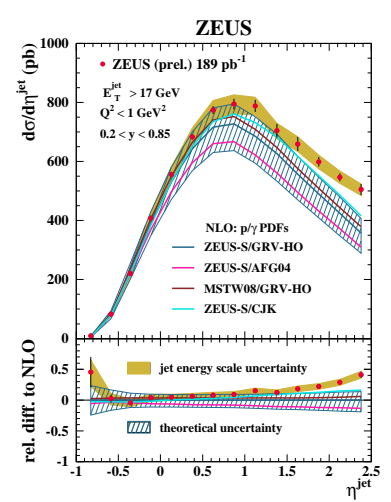

(b)

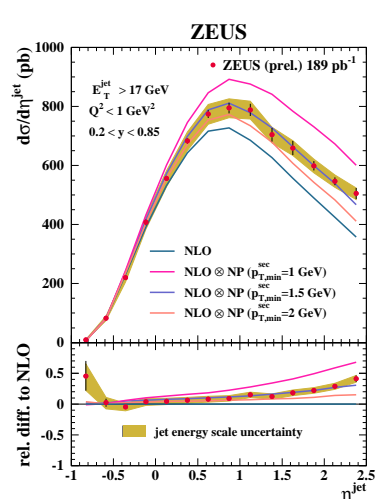

(c)

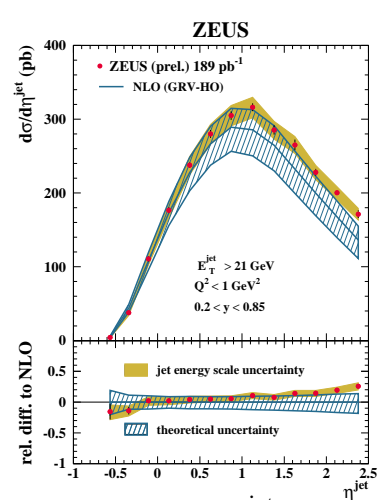

Figure 9: Inclusive-jet cross section as a function $\eta^{\text {jet }}$ for (a) and (b) $E_{T}^{\text {jet }}>17 \mathrm{GeV}$ and (c) $E_{T}^{\text {jet }}>21 \mathrm{GeV}$.

which shows the cross section as a function of $\eta^{\text {jet }}$ for $E_{T}^{\text {jet }}>21 \mathrm{GeV}$.

The dijet cross sections in photoproduction as functions of $M^{\mathrm{jj}}$ and $\left|\cos \theta^{*}\right|$ are well suited to test the underlying dynamics. The invariant mass is especially sensitive to the form of the matrix elements and $\theta^{*}$, which coincides with the parton-parton scattering angle in the dijet centre-ofmass system, is sensitive to the spin of the exchanged particle; a different behaviour is predicted for resolved and direct processes which arises from the dominating exchanged particle: gluons for resolved and quarks for direct processes. Figures $8 \mathrm{c}$ and $8 \mathrm{~d}$ show the measured cross sections as functions of these two observables [14]. The NLO calculations give a very good description of the data. No significant deviation with respect to QCD is observed, specially at high masses.

Testing pQCD with jets requires infrared- and collinear-safe jet algorithms. Up to now, only the $k_{T}$ algorithm fulfilled these requirements at all orders. This algorithm has been tested extensively at HERA and a good description of the data was obtained for different jet radii [19] with similar precision. Therefore, the $k_{T}$ algorithm has a good performance with small theoretical uncertainties and small hadronisation corrections. Recently, new infrared- and collinear-safe jet algorithms, namely the anti- $k_{T}$ [20] and SIScone [21], have become available. Studies have been done at HERA in the NC DIS and photoproduction regimes to validate these algorithms. The performance of the anti- $k_{T}$ and SIScone algorithms has been tested in hadron-induced but well understood reactions. The measurements based on the new algorithms have been compared to those based on the $k_{T}$, a comparison between data and theory has been performed and studies of the theoretical uncertainties and hadronisation corrections have been done.

The theoretical uncertainties for these new jet algorithms have been studied by ZEUS in the DIS [22] and photoproduction [23] regimes. The uncertainties from the PDFs and the value of $\alpha_{s}$ are very similar for all three algorithms. The uncertainty from terms beyond NLO and the modelling of the parton shower are very similar for the $k_{T}$ and anti- $k_{T}$, but slightly larger for the SIScone algorithm. The ZEUS Collaboration has measured the inclusive-jet cross sections as functions of the jet transverse energy using the three jet algorithms [22, 23] (see Fig. 10). The shape and normalisation of the measured and predicted cross sections are very similar. The experimental uncertainties are similar. The data are very well described by the NLO calculations. The hadronisation-correction factor applied to the calculations are similar for the $k_{T}$ and anti- $k_{T}$ and somewhat bigger for the SIScone algorithm. To study in more detail the performance of the new algorithms, ZEUS has measured the ratios of the cross sections based on the three jet algorithms. Figures $10 \mathrm{e}$ and $10 \mathrm{f}$ show the measured ratios for anti- $k_{T} / k_{T}$, SIScone $/ k_{T}$ and anti- $k_{T} /$ SISCone as 
(a)

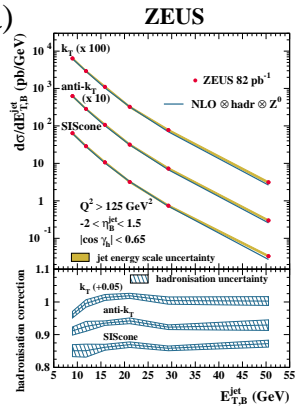

(b)

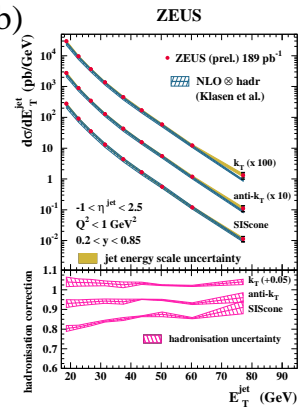

(c)

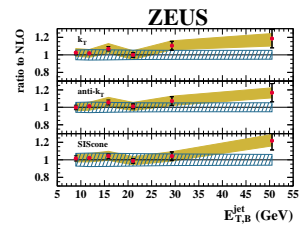

(d)

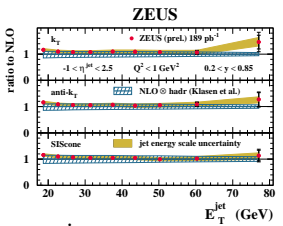

(e)

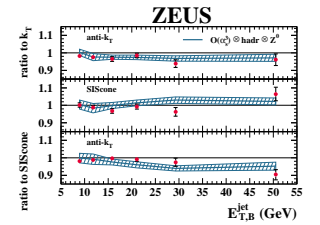

(f)

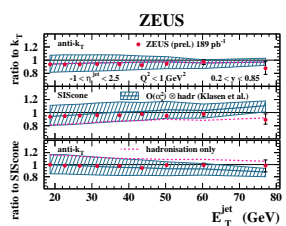

Figure 10: Inclusive jet cross sections as functions of (a) $E_{T, \mathrm{~B}}^{\mathrm{jet}}$ in NC DIS and (b) $E_{T}^{\mathrm{jet}}$ in photoproduction based on different jet algorithms. (c) and (d) the relative difference between the data of (a) and (b) and the NLO calculations. Ratios of the cross sections anti- $k_{T} / k_{T}$, SIScone $/ k_{T}$ and anti- $k_{T} /$ SIScone as functions of (e) $E_{T, \mathrm{~B}}^{\mathrm{jet}}$ in NC DIS and (f) $E_{T}^{\mathrm{jet}}$ in photoproduction.

functions of $E_{T}^{\text {jet }}$. The measured cross sections show differences below $3.6 \%$ at low $E_{T}^{\text {jet }}$, which increase to $10 \%$ at high $E_{T}^{\mathrm{jet}}$. In photoproduction, the cross section based on the anti- $k_{T}$ has the same shape as the $k_{T}$ but it is $6 \%$ lower. The SIScone cross section displays slightly different shape than the $k_{T}$ and anti- $k_{T}$. For NC DIS, QCD predictions up to $\mathscr{O}\left(\alpha_{s}^{3}\right)$ are available, whereas for photoproduction, the calculations are only $\mathscr{O}\left(\alpha_{s}^{2}\right)$. The predictions give a good description of the measured ratios. The uncertainty due to higher orders in the $\mathscr{O}\left(\alpha_{s}^{3}\right)$ calculation is reduced and so the dominant uncertainty is that due to the QCD-cascade modelling. These results demonstrate the ability of pQCD with up to four partons in the final state to account adequately for the differences between jet algorithms. In addition, values of $\alpha_{s}\left(M_{Z}\right)$ were extracted from the measured cross sections in NC DIS and photoproduction using the three jet algorithms to quantify their performance. These determinations are consistent with each other and have a similar precision.

\section{Summary and conclusions}

Jet physics at HERA continues providing precision measurements towards the understanding of QCD and the improvement of the determination of the proton and photon PDFs. New jet measurements were presented which were optimised to constrain further the PDFs and test the performance of new jet algorithms. In addition, precise values of $\alpha_{s}\left(M_{Z}\right)$ were extracted from jet production in different regimes; Fig. 11a shows a summary of the determinations presented together with the world [24] and HERA [25] averages. The values are consistent with each other and the world average. In addition, a precise determination of the running of $\alpha_{s}$ was obtained over a wide range of the scale (see Figs. 11b and 11c).

\section{References}

[1] ZEUS Collaboration, S. Chekanov et al., Eur. Phys. J. C 42 (2005) 1.

[2] ZEUS Collaboration, H. Abramowicz et al., Eur. Phys. J. C 70 (2010) 965.

[3] H1 Collaboration, F.D. Aaron et al., Eur. Phys. J. C 67 (2010) 1.

[4] S. Catani et al., Nucl. Phys. B 406 (1993) 187.

[5] S.D. Ellis and D.E. Soper, Phys. Rev. D 48 (1993) 3160. 

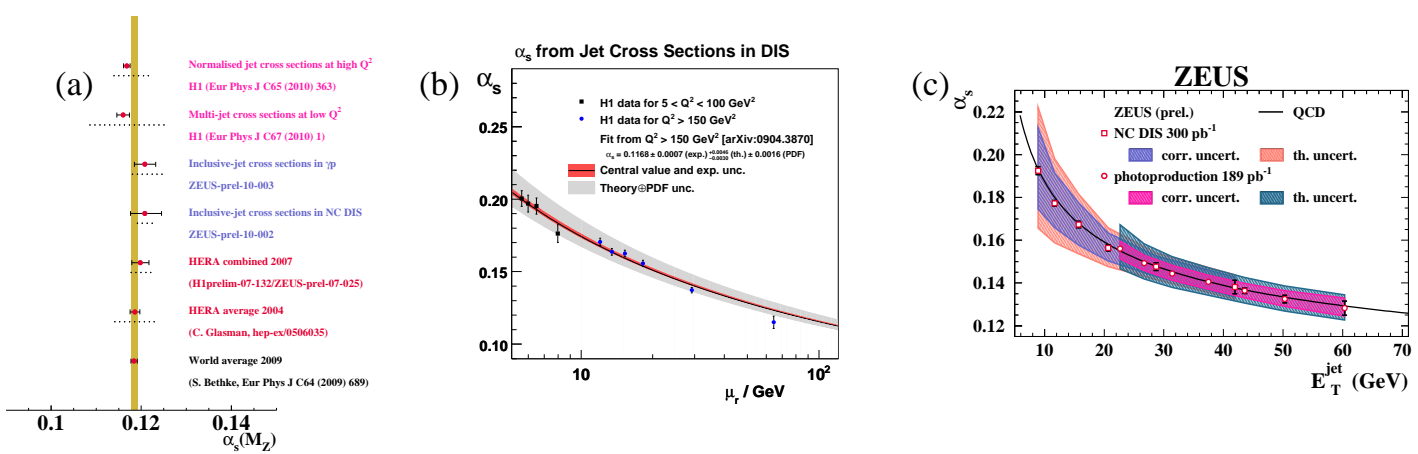

Figure 11: (a) Summary of $\alpha_{s}\left(M_{Z}\right)$ determinations at HERA; (b) $\alpha_{s}$ as a function of the scale (H1 Collaboration); (c) $\alpha_{s}$ as a function of $E_{T}^{\mathrm{jet}}$ (ZEUS Collaboration).

[6] Z. Nagy and Z. Trocsanyi, Phys. Rev. Lett. 87 (2001) 082001.

[7] W.K. Tung et al., JHEP 0702 (2007) 053.

[8] P.M. Nadolsky et al., Phys. Rev. D 78 (2008) 013004.

[9] ZEUS Collaboration, H. Abramowicz et al., ZEUS-prel-10-002.

[10] S. Catani and M.H. Seymour, Nucl. Phys. B 485 (1997) 291.

[11] ZEUS Collaboration, S. Chekanov et al., Phys. Rev. D 67 (2003) 012007.

[12] ZEUS Collaboration, H. Abramowicz et al., ZEUS-prel-10-003.

[13] M. Klasen, T. Kleinwort and G. Kramer, Eur. Phys. J. Direct C 1 (1998) 1.

[14] ZEUS Collaboration, H. Abramowicz et al., ZEUS-prel-10-014.

[15] H1 Collaboration, F.D. Aaron et al., Eur. Phys. J. C 65 (2010) 363.

[16] P. Aurenche, M. Fontannaz and J.Ph. Guillet, Eur. Phys. J. C 44 (2005) 395.

[17] M. Glück, E. Reya and A. Vogt, Phys. Rev. D 45 (1992) 3986;

M. Glück, E. Reya and A. Vogt, Phys. Rev. D 46 (1992) 1973.

[18] F. Cornet, P. Jankowski and M. Krawczyk, Phys. Rev. D 70 (2004) 093004.

[19] ZEUS Collaboration, S. Chekanov et al., Phys. Lett. B 649 (2007) 12.

[20] M. Cacciari, G.P. Salam and G. Soyez, JHEP 0804 (2008) 063.

[21] G.P. Salam and G. Soyez, JHEP 0705 (2007) 086.

[22] ZEUS Collaboration, H. Abramowicz et al., Phys. Lett. B 691 (2010) 127.

[23] ZEUS Collaboration, H. Abramowicz et al., ZEUS-prel-10-015.

[24] S. Bethke, Eur. Phys. J. C 64 (2009) 689.

[25] C. Glasman, Proc. of the 13th International Workshop on Deep Inelastic Scattering (Madison, USA, 2005) and hep-ex/0506035;

C. Glasman, Proc. of the HEP2007 International Europhysics Conference on High Energy Physics (Manchester, England, 2007) and hep-ex/0709.4426. 